\title{
PATHOLOGICAL EVALUATION OF DORSAL ROOT GANGLIA AND SPINAL CORD AND SCIATIC NERVE FOLLOWING A CHRONIC CONSTRICTION INJURY OF THE SCIATIC NERVE IN RATS
}

Al-Shimaa E Elsayed ${ }^{1}$, Walaa $F$ Awadin ${ }^{1}$, Marwa E Abass ${ }^{2}$, and Mohamed F Hamed $^{1}$

${ }^{1}$ Department of Pathology, Faculty of Veterinary Medicine, Mansoura University, Mansoura 35516, Egypt.

${ }^{2}$ Department of Surgery, Anesthesiology and Radiology, Faculty of Veterinary Medicine, Mansoura University, Mansoura 5516, Egypt.

*Correspondence to: Al-Shimaa E Elsayed; Tel. 01018912584, Email: alshimaaelhefny@gamil.com

\section{ABSTRACT}

Objective: To study pathological changes on spinal cord and dorsal root ganglia (DRG) and sciatic nerve itself after sciatic nerve injury in rats

Design: Randomized experimental study

Procedure: Rats were randomly divided into two main groups. Control group (25 rats) without any treatment and treated group $(n=50)$ with induced chronic constriction injury (CCI) of sciatic nerve. The rates of the treated groups were euthanized at intervals, 25 rats after two weeks and the other 25 rats at 4 weeks after injury. Histopathological examinations of sciatic nerve, spinal cord and DRG for each group were done.

Results: The sensory neurons of the DRG displayed chromatolysis at two weeks post-injury, while chromatolysis, satellitosis and microgliosis were evident at four weeks post-injury. Wallerian degeneration in the sciatic nerve, and inflammatory cells recruitment with glial cells proliferation in spinal cord, was consistent at four weeks of CCI.

Conclusion and clinical relevance: We concluded that DRG showed deleterious pathological alterations in the sensory neurons following sciatic nerve injury in rats.

Keywords: Chronic constriction injury, Dorsal root ganglia, Spinal 
cord, Rats 


\section{INTRODUCTION}

Chronic constriction injury of the sciatic nerve (CCI) is a model of peripheral nerve injury (PNI) that was established in 1988 [1]. It is considered as a means to produce neuropathic pain in laboratory animal models [2]. This model produces a pathological condition that is quite similar to human peripheral nerve injury with its consequent behavioral manifestations [3]. Thirty percent of PNI arose from lacerations by sharp objects and long bone fractures [4]. The remaining penetrating injuries, crush, ischemia, traction, electric shock and vibration play a role as well [5].

The dorsal root ganglia (DRG) are found between the dorsal root and the spinal nerve. It contains pseudo unipolar neurons that carry sensory information from the peripheral receptors to the central nervous system for a response [6].

After nerve injury or inflammation, the sensory neurons in the DRG may become an important cause of increased nociceptive signaling through increased neuronal excitability and generation of ectopic discharges [7]. Many factors have to be taken into consideration when trying to predict the outcome of peripheral nerve repair, including type, location and extent of nerve injury; timing of surgery; type of repair; proper alignment of fascicles; surgical technique and patient comorbidities [8].

The present study has investigated to shed light on the pathological changes and the secondary anterograde degeneration of the rat sciatic nerve fiber, dorsal root ganglion and spinal cord after chronic constriction injury. 


\section{MATERIAL AND METHODS}

\subsection{Animals}

This study was performed on Seventy-five healthy male albino rats weighing 190-220 g and aged 2-3 months. Rats were kept in cages (4 rats/cage) on a sawdust bedding under controlled laboratory conditions (12 h light/dark cycle, lights on from 8:00 am) with temperature $20^{\circ} \mathrm{C}$, air humidity $55-60 \%$ and free access to food and water. Five days before the experiments, they were daily weighted, handled gently for 5 minutes, and placed in the test room for 2 hours (from 09:00 AM to 11:00 AM), where they were left to become accustomed to the surrounding environment. All experiments performed between 09:00 AM and 11 :00 AM.

\subsection{Experimental design}

Seventy-five healthy male albino rats were randomly divided into two main groups. First group $(n=25)$ was a control negative group in which the rats were kept for month without surgery. In the second treated group $(n=50)$, rats were subjected to CCI. The rates of the treated groups were euthanized at 2 intervals, whereas 25 rats were euthanized at two weeks post CCI, and, the other 25 rats were euthanized at four weeks post CCI of the sciatic nerve.

\subsection{Surgical procedures}

A CCI of the sciatic nerve was performed according to [1], under intraperitoneal injection of medetomidine hydrochloride $0.4 \mathrm{mg} / \mathrm{kg}$ (Domitor 1\%, Pfizer, Seixal, Portugal) and ketamine hydrochloride 60 $\mathrm{mg} / \mathrm{kg}$ (Ketamax 5\%, Troikaa, Gujarat, India;[9]. 
Briefly, the left hind limb was aseptically prepared then a skin incision was made 3-4 mm below and parallel to the femur. A blunt dissection was made between the gluteus superficialis and the biceps femoris muscles using blunt curved scissors then widening of the gap between the two muscles by self-wound retractor to clear visualization of the sciatic nerve. Using curved blunt-tipped forceps and micro-scissors, approximately $10 \mathrm{~mm}$ of the sciatic nerve was gently freed (proximal to the sciatic trifurcation) from the surrounding connective tissue.

Four ligatures were placed around and proximal to the trifurcation of the sciatic nerve with 1-mm spacing using chromic gut 4.0 (absorbable suture, Sharpoint ${ }^{\circledR}$, USA). Constriction of the nerve was immediately stopped if a brief twitch was observed to prevent arresting of the epineural blood flow over tightening the ligatures and to avoid axotomy and autotomy (self-mutilation) both unwanted side-effects which preclude successful pain hypersensitivity testing [10].

The muscles overlying the nerve were sutured with two rows of a simple continuous pattern chromic using gut ligature (3-0) (absorbable suture, Sharpoint ${ }^{\circledR}$, USA). The wound was irrigated with normal saline then the skin incision was sutured with simple interrupted pattern using surgical suture silk 3.0 (non-absorbable, braided suture, Ghatwary medical, Egypt). Betadine dressing was used postoperatively

Immediately following surgery, the rats were placed on a warmed pad, filter-topped recovery cage. A systemic anti-inflammatory and antibiotic drugs were intramuscularly injected once daily for 5 days (meloxicam $6 \mathrm{mg} / \mathrm{kg}$; Anticox $\prod, 5 \mathrm{mg} / \mathrm{ml}$, Adwai, 10th of Ramadan city, Egypt and cefotaxime $60 \mathrm{mg} / \mathrm{kg}$; Taximodel $1 \mathrm{gm}$, Pharco, Egypt). Besides, a local topical antibiotic spray neomycin, bacitracin (Bivatracin, Kafrelsheikh Vet. Med. J. Vol. 17 No. 1 (2019) 
Acadima international, Egypt) was applied once daily until healing of the wound. The skin stitches were removed after ten days.

\subsection{Macroscopic and Microscopic finding}

All macroscopic findings were recorded for month post CCI.

After perfusion, specimens from sciatic nerve, spinal cord and dorsal root ganglia were obtained from each group and submitted to Pathology Department, Faculty of Veterinary Medicine, Mansoura University in $10 \%$ neutral buffered formalin. (Formalin fixed tissues specimens were dehydrated in ascending grades of alcohol concentrations, cleared in xylene, and embedded in molten paraffin. Five $\mu \mathrm{m}$ thick paraffin sections were cut with a microtome and again cleared in grades of xylene, dehydrated in graded alcohol concentrations then stained with hematoxylin and eosin (H\&E) [11].

\section{RESULTS}

\subsection{Macroscopic findings}

The animal arches its back upwards with rough hair coat and porphyrin staining (read tears around nose and eye of rats). As well as, abnormal gait and posture in the affected limb which appear as dragging of the limb or standing on knee joint limb not toe and hind paw shrinkage (Fig 1).

\subsection{Microscopic findings}

Dorsal root ganglia in the control group have clusters of cell bodies that varied in size to large, intermediate and small neurons (Fig. 2A). After 2 weeks of chronic sciatic nerve constriction, DRG showed alterations in normal histological architecture represented in edema and 
disorganization of neurons. Neurons had central chromatolysis with nucleus pushed peripherally especially in the small sized ones (Fig. 2B). After 4 weeks of sciatic nerve injury, DRG showed contracted neurons with marked central chromatolysis particularly in small and medium sized neurons. Proliferation of microglia (microgliosis) and satellite cells (satellitosis) were also seen within the DRG (Fig. 2C\&D).

In the control group, sciatic nerve exhibited normal nerve fibers with normal Schwan cells and normal epineurium (Fig. 3A). After 2 weeks of injury, sciatic nerve showed edema in perineurium, necrotic Schwan cells and degenerated nerve fibers (Fig. 3 B). After 4 weeks of injury, sciatic nerve showed Wallerian degeneration and axonal spheroids (Fig. 3C). Secondary demyelination with presence of foamy macrophages engulfed myelin was also observed (Fig. 3D) Peripheral granulomatous neuritis represented by aggregation of macrophages and epithelioid cells was additionally recorded

Spinal cord of the control group was consisted of peripheral white matter and inner gray matter with normal central canal, neurons, astrocytes and microglia (Fig. 4A). while after 2 weeks of sciatic nerve injury showed abscessation in white matter. Aggregation of neutrophils within the white matter and at the junction between white and gray matter was also seen. Marked astrocytosis and astrogliosis in gray matter was observed as well (Fig. 4B). After 4 weeks of sciatic nerve injury, spinal cord neurons showed central chromatolysis with peripherally dispersed Nissl granules and slightly eccentric nucleus. Activated microglia cells was also seen (Fig. 4C). 


\section{DISCUSSION}

The present study investigated the pathological alterations in DRG, sciatic nerve and spinal cord following chronic constriction injury of the sciatic nerve in rat model. Microscopic examination showed that the most affected neurons after sciatic nerve injury were the small sized ones. These findings may indicate that the small dark populations of neurons are comprised mostly of primary sensory ones. The latter convey nociceptive signals, so these neurons might play a key role in neuropathic hypersensitivity $[\mathbf{1 2}, \mathbf{1 3}]$

The central chromatolysis particularly in small and medium sized neurons was the most consistent lesion after sciatic nerve injury. The lesion severity increased with increasing time of exposure. Central chromatolysis is a histopathologic change seen in the cell body of a neuron. This process occurs after an injury to the neuron and is sustained and/or irreparable. Central chromatolysis is characterized by tumefaction of cell body and Nissl bodies are dissolved from the cell center and peripherally displaced along with the nucleus [14]. It is an axonal reaction where changes appear to reflect reversible changes in cell metabolism that include ischemia; these changes are interpreted as a state of heightened metabolic activity that favors axonal regeneration $[15,16]$. Chromatolysis and inflammation are characteristic of neuronal cells with compromised microcirculation that can disrupt communication in many portions of the central nervous system [17]. Chromatolysis also causes degeneration of myelin sheaths and neuronal death in both the peripheral nerves and the central nervous system. These and associated conduction block markedly aggravate damage and neurological dysfunction in SCI [18]. In this study, the prominent histopathological alterations were 
proliferation of microglia (microgliosis) and satellite cells (satellitosis). These findings are consistent with the data of the previous approaches [19]. Satellite cells respond to damage of sensory neurons in DRG, so these cells may play a role in the pathological changes in the ganglia [19]. Chronic sciatic nerve compression was characterized by secondary demyelination as mentioned by [20]. The spinal cord inflammatory cells recruitment and glial cells proliferation and activation were hall markers in the most of neuropathic pain models [21-23]

\section{CONCLUSION}

DRG and spinal cord neurons showed deleterious pathological alterations in the sensory neurons that increased with increasing time of exposure following sciatic nerve injury.

\section{Conflict of interest statement}

The authors declare that there is no any conflict of interest in the current research work

\section{Animal ethics committee permission}

The current research work is permitted to be executed according to standards of animal research committee in Faculty of Veterinary Medicine, Mansoura University.

\section{Author contributions}

Walaa F Awadin designed the study, interpreted the data, and drafted the manuscript. Mohamed F Hamed was involved in collection of data and also contributed in manuscript preparation. Marwa E Badawy performed the the surgical experiment and Al-Shimaa E Elsayed took part in preparing and critical checking of this manuscript. 


\section{Figure legends:}

Fig.(1): Red tears (porphria), rough erected hair and arched back with prescience of edema and flexion of fingers.

Fig. (2): Microscopic pictures of H\&E stained DRG displays (A) normal neurons (arrow) with normal satellite cells (arrow head), (B) central chromatolysis with nucleus pushed to peripherally (arrow) and enlarged satellite cells with mild macrophagic recruitment (arrowhead), (C\&D) small sized neuron with central chromatolysis (arrow) and macrophagic infiltration (arrowhead; 400x)

Fig. (3): Microscopic pictures of H\&E stained sciatic nerve showing (A) normal nerve fibers with normal Schwan cell (arrow) and normal epineurium. (100x), (B) edema in perineurium, necrotic Schwan cells and degenerated nerve fibers (arrow) 2 weeks of injury, (100x), (C) Wallerian degeneration and axonal spheroids (arrow) 4 weeks of injury (400x), (D) secondary demyelination with presence of foamy macrophages (arrow) (400x), (E) granulomatous peripheral neuritis represented by aggregation of macrophages and epithelioid cells (arrow; 400x).

Fig. (4): Microscopic pictures of $H \& E$ stained spinal cord displays (A) normal neurons (arrow), astrocytes (arrow head) and normal microglia (red arrow), (B) neutrophilic infiltrate (arrow head) and degeneration of nerve fibers at white matter, $(\mathbf{C})$ central chromatolysis with dispersed Nissl granules at the neuron peripherally and slightly eccentric nucleus (arrow) and activated microglia cells (arrow head; 400x). 

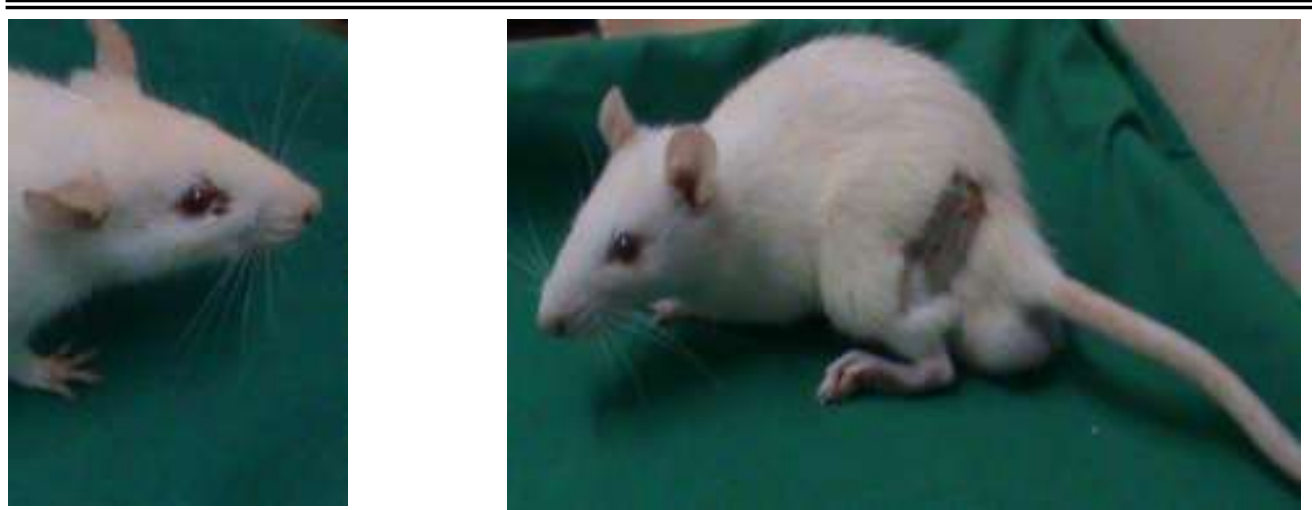

Fig.(1): Red tears (porphria), rough erected hair and arched back with prescience of edema and flexion of fingers in rats after CCI.

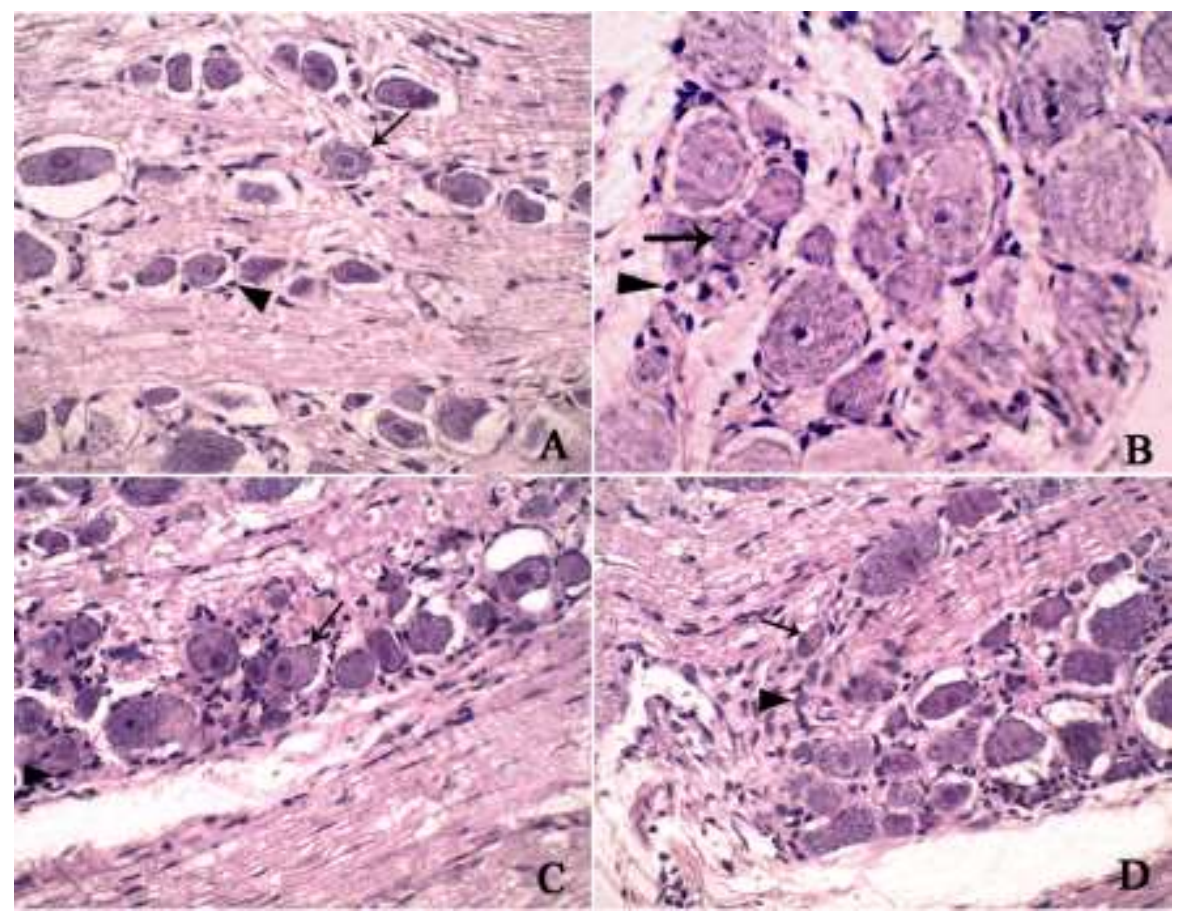

Fig. (2): Microscopic pictures of H\&E stained DRG displays (A) normal neurons (arrow) with normal satellite cells (arrow head), (B) central chromatolysis with nucleus pushed to peripherally (arrow) and enlarged satellite cells with mild macrophagic recruitment (arrowhead), (C\&D) small sized neuron with central chromatolysis (arrow) and macrophagic infiltration (arrowhead; 400x) 


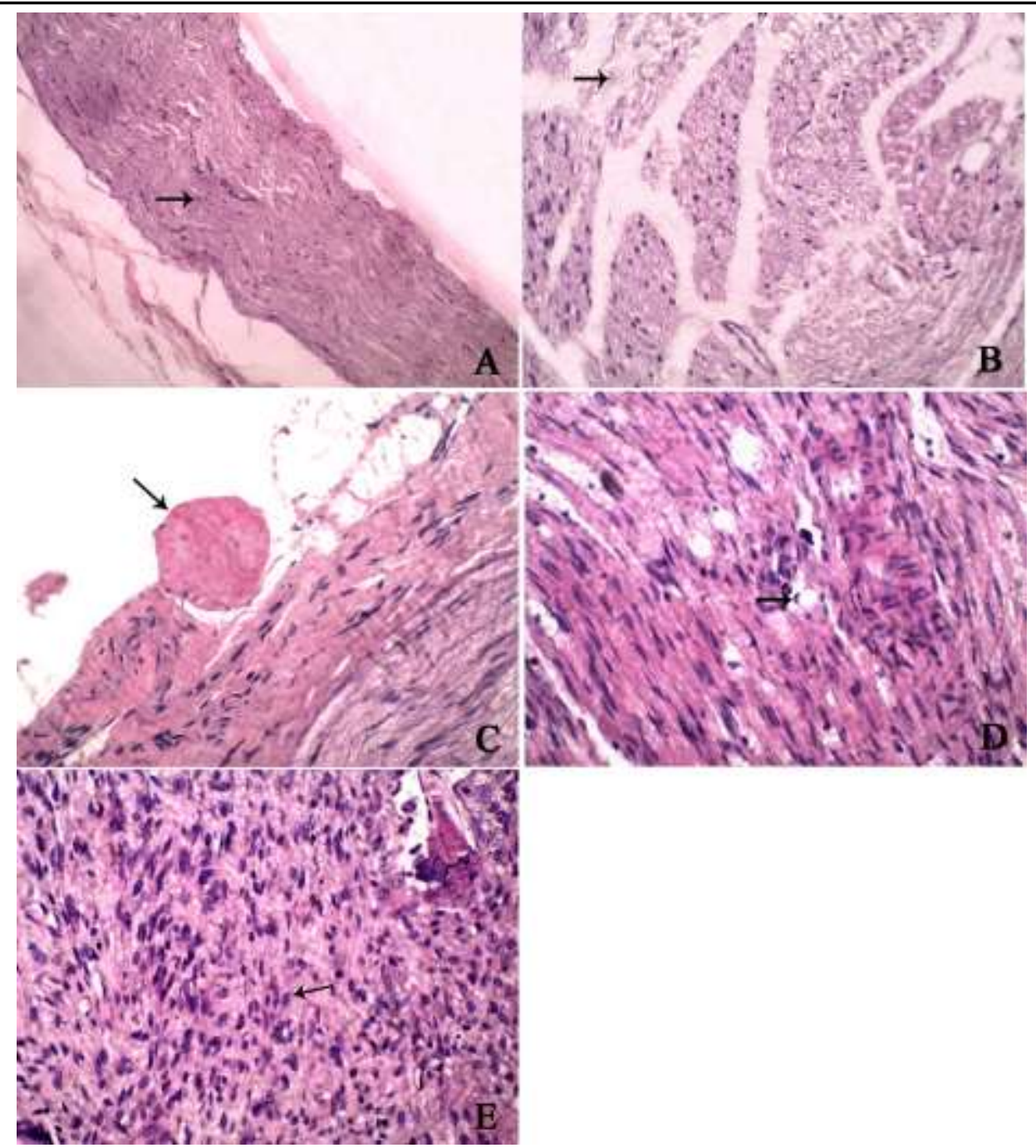

Fig. (3): Microscopic pictures of $H \& E$ stained sciatic nerve showing (A) normal nerve fibers with normal Schwan cell (arrow) and normal epineurum. (100x), (B) edema in perineurum, necrotic Schwan cells and degenerated nerve fibers (arrow) 2 weeks from injury, (100x), (C) Wallerian degeneration and axonal spheroids (arrow) 4 weeks from injury (400x), (D) secondary demyelination with presence of foamy macrophages (arrow) (400x), (E) granulomatous peripheral neuritis represented by aggregation of macrophages and epithelioid cells (arrow; 400x). 


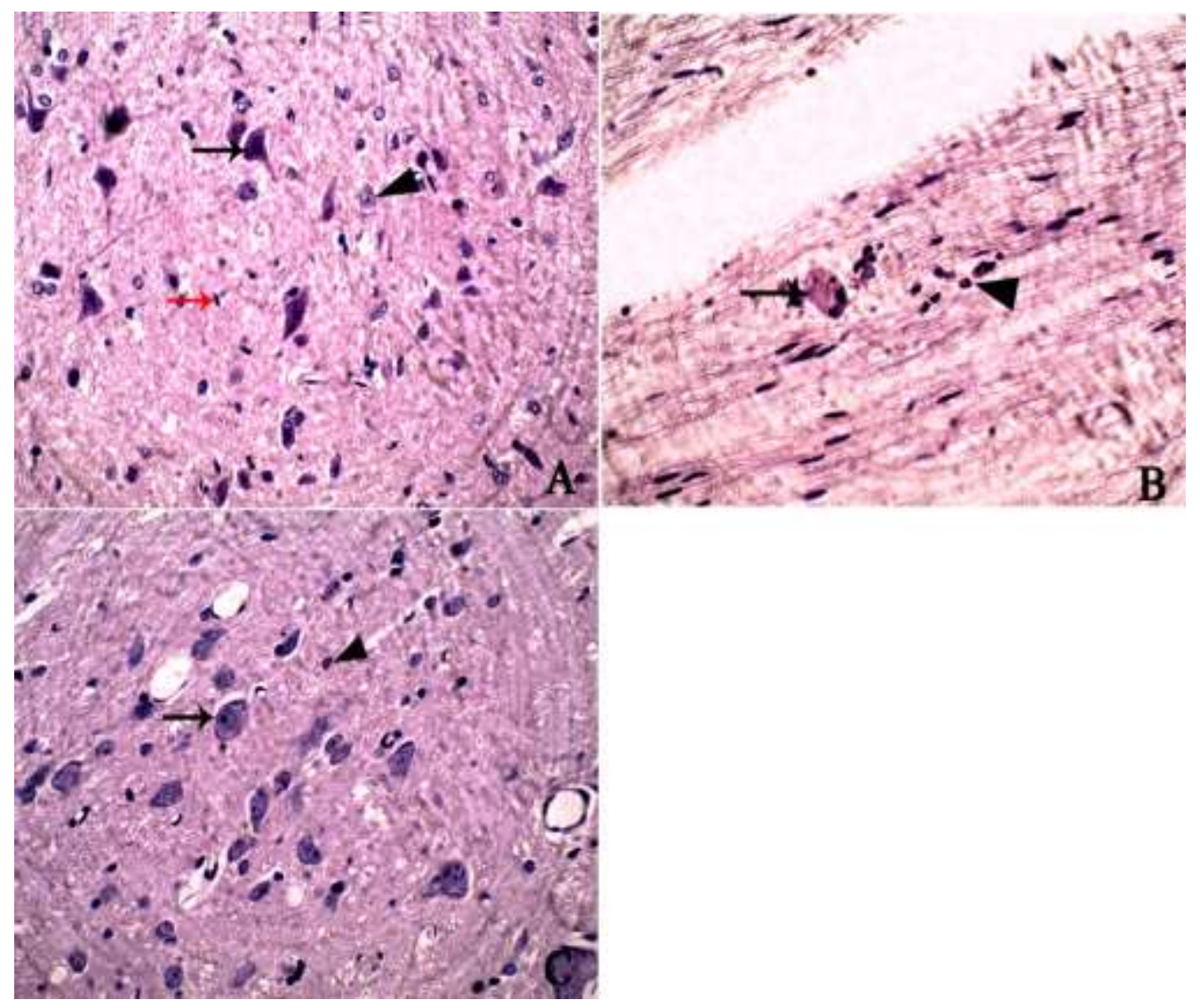

Fig. (4): Microscopic pictures of H\&E stained spinal cord displays (A) normal neurons (arrow), astrocytes (arrow head) and normal microglia (red arrow), (B) neutrophilic infiltrate (arrow head) and degeneration of nerve fibers at white matter, $(\mathbf{C})$ central chromatolysis with dispersed Nissl granules at the neuron peripherally and slightly eccentric nucleus (arrow) and activated microglia cells (arrow head; 400x). 


\section{REFERENCES}

- [1] Bennett GJ, Xie Y-K. A peripheral mononeuropathy in rat that produces disorders of pain sensation like those seen in man. Pain 1988;33:87-107.

- [2] Zhang Y, Zhan Y, Han N, Kou Y, Yin X, Zhang P. Analysis of temporal expression profiles after sciatic nerve injury by bioinformatic method. Scientific reports 2017;7:9818.

- [3] Zhi M-J, Liu K, Zheng Z-L, He X, Li T, Sun G, et al. Application of the chronic constriction injury of the partial sciatic nerve model to assess acupuncture analgesia. Journal of pain research 2017;10:2271.

- [4] Stanec S, Tonković I, Stanec Z, Tonković D, Džepina J. Treatment of upper limb nerve war injuries associated with vascular trauma. Injury 1997;28:463-8.

- [5] Robinson LR. Traumatic injury to peripheral nerves. Muscle \& Nerve: Official Journal of the American Association of Electrodiagnostic Medicine 2000;23:863-73.

- [6] Pope JE, Deer TR, Kramer J. A systematic review: current and future directions of dorsal root ganglion therapeutics to treat chronic pain. Pain Medicine 2013;14:.96-1477

- [7] Xie W-R, Deng H, Li H, Bowen T, Strong J, Zhang J-M. Robust increase of cutaneous sensitivity, cytokine production and sympathetic sprouting in rats with localized inflammatory irritation of the spinal ganglia. Neuroscience 2006;142:809-22. 
- [8] Siemionow M, Brzezicki G. Current techniques and concepts in peripheral nerve repair. International review of neurobiology 2009;87:141-72.

- [9] Abass M, Mosbah E, Rizk A, Karrouf G, Zaghloul A. Synergistic efficacy of tramadol and meloxicam on alleviation of pain and selected immunological variables after sciatic nerve ligation in rats. International Journal of Veterinary Science and Medicine 2014;2:1420.

- [10] Austin PJ, Wu A, Moalem-Taylor G. Chronic constriction of the sciatic nerve and pain hypersensitivity testing in rats. JoVE (Journal of Visualized Experiments) 2012:e3393.

- [11] Bancroft JD, Gamble M. Theory and practice of histological techniques: Elsevier health sciences; 2008.

- [12] Woolf CJ, Salter MW. Neuronal plasticity: increasing the gain in pain. science 2000;288:1765-8.

- [13] Zimmermann M. Pathobiology of neuropathic pain. European journal of pharmacology 2001;429:23-37.

- [14] Callegari DC, de Paula Posso I, Galupo MTG, Wroclawski ER. Acute ketoprofen neurotoxicity in spinal cord of rats. EINSTEINSAO PAULO 2008;6:82-5.

- [15] Errando CL, Sifre C, Moliner S, Valá JC, Gimeno O, Mínguez $\boldsymbol{A}$, et al. Subarachnoid ketamine in swine - pathological findings after repeated doses: acute toxicity study. Regional anesthesia and pain medicine 1999;24:146-52. 
- [16]Kreutzberg $\boldsymbol{G W}$. Principles of neuronal regeneration. Mechanisms of Secondary Brain Damage in Cerebral Ischemia and Trauma: Springer; 1996. p. 103-6.

- [17] Guyton AC, Hall J. Dietary balances; regulation of feeding; obesity and starvation; vitamins and minerals. Textbook of Medical Physiology (Guyton AC, Hall JE, eds) Elsevier Saunders Inc Philadelphia, PN 2006:876.

- [18] McTigue DM. Potential Therapeutic Targets for PPARII after Spinal Cord Injury. PPAR research 2008;2008.

- [19] Hanani M. Satellite glial cells in sensory ganglia: from form to function. Brain research reviews 2005;48:457-76.

- [20] MacKinnon DP, Lockwood CM, Hoffman JM, West SG, Sheets $\boldsymbol{V}$. A comparison of methods to test mediation and other intervening variable effects. Psychological methods 2002;7:83.

- [21] Colburn R, Rickman A, DeLeo J. The effect of site and type of nerve injury on spinal glial activation and neuropathic pain behavior. Experimental neurology 1999;157:289-304.

- [22] Garrison C, Dougherty PM, Kajander K, Carlton S. Staining of glial fibrillary acidic protein (GFAP) in lumbar spinal cord increases following a sciatic nerve constriction injury. Brain research 1991;565:1-7.

- [23] Hu B, Doods H, Treede R-D, Ceci A. Depression-like behaviour in rats with mononeuropathy is reduced by the CB2-selective agonist GW405833. PAIN® 2009;143:206-12. 